Viktor Schell*, Georg Böttcher, Leon Budde, M. Geraldine Zuniga, Thomas Lenarz and Thomas S. Rau

\title{
A software for online monitoring of orientation-compensated forces during $\mathrm{Cl}$ insertion
}

\begin{abstract}
The electrode array insertion is a critical point during CI surgery and should be performed as gently as possible to preserve residual hearing. In order to measure occurring forces, an insertion tool with an integrated force sensor and an inertial measurement unit (IMU) is being developed. The weight of the electrode holder and the sensor add an unknown offset to the measured forces, depending on the tool orientation. To address this problem, a software which calculates the orientation-induced error and computes a corrected force was developed.
\end{abstract}

The software was written in C++ using the library Qt 5.12.9. For maximization of the computing frequency, the data acquisition of both sensors and the monitoring was parallelized. An algorithm was developed to calculate the error caused by the electrode holder and sensor. For this purpose both weights were determined in a calibration procedure and merged with the provided IMU data. The evaluation was done in two test series (each $n=5$ ) with different initial tool orientations. To this end a stepwise $360^{\circ}$ rotation around the horizontal axis was performed, while recording the corrected forces.

The developed software allows a computing frequency up to $100 \mathrm{~Hz}$ with a latency of $10 \mathrm{~ms}$ for the online monitoring of the processed data. The evaluation of the corrected force shows a residual error of $0.347 \mathrm{mN} \pm 0.325 \mathrm{mN}$ for the first and $0.176 \mathrm{mN} \pm 1.971 \mathrm{mN}$ for the second test series.

With the created algorithm, the impact of the extra weight on the sensor can be almost fully equalized. The highly responsive software offers a new possibility to process

\footnotetext{
${ }^{*}$ Corresponding author: Viktor Schell: Hannover Medical School, Department of Otolaryngology, Carl-Neuberg-Strasse 1, 30625 Hanover, Germany, schell.viktor@mh-hannover.de Georg Böttcher: Hannover Medical School, Department of Otolaryngology, Hanover, Germany

Leon Budde: Leibniz University Hannover, Institute of Mechatronic Systems, Hanover, Germany

M. Geraldine Zuniga, Thomas Lenarz, Thomas Rau: Hannover Medical School, Department of Otolaryngology and Cluster of Excellence EXC 2177/1 “Hearing4all", Hanover, Germany
}

insertion forces and provide feedback to surgeons. Determining the influence of the tool orientation on the corrected forces is the subject of future researches.

Keyw ords: CI insertion, software, orientation compensated force, compensated force.

https://doi.org/10.1515/cdbme-2021-2025

\section{Motivation}

Cochlear implant (CI) systems can be divided in two parts: an external sound processor and the implanted part that receives auditory signals and transmits them to the cochlear nerve. To enable this latter transmission, an electrode array (EA) is inserted into the cochlea during CI surgery. Studies have shown that a gentle insertion reduces the probability of intracochlear trauma [1,2], which is connected to the loss of residual hearing $[3,4]$.

The magnitude of forces occurring during EA insertion is only perceived by the surgeon's fingertips but cannot yet be objectively determined. For this reason, a force sensitive insertion tool is under development. A force sensor within the tool is connected to an electrode holder, which enables the measurement of forces acting axially along the EA. Initial tests have shown that the unloaded insertion tool recorded orientation-dependent forces caused by the weight of the electrode holder and the sensor. Therefore, an inertial measurement unit (IMU) was integrated into the insertion tool to allow compensation of this orientation-dependent force component.

The purpose of this work was to establish a method to clear the measured signal from perturbations caused by the weight of the electrode holder and the sensor. We present a software that both calculates orientation-dependent errors from the measured signal and provides visual feedback on the corrected force. 


\section{Material and Methods}

\subsection{Insertion tool prototype}

The basis for force measurements during EA insertions is a manual insertion tool that is currently being developed inhouse (see Figure 1). It is a small device with an embedded one dimensional force sensor (KD24s 2N, ME-Meßsysteme $\mathrm{GmbH}$, Hennigsdorf, Germany) in combination with a GSV-8DS measurement amplifier (ME-Meßsysteme GmbH, Hennigsdorf, Germany) and an inertial measurement unit (BNO055, Bosch Sensortec GmbH, Reutlingen, Germany). An electrode holder coaxially holding the EA is connected to the force sensor, ensuring the direct transmission of forces in the insertion direction.

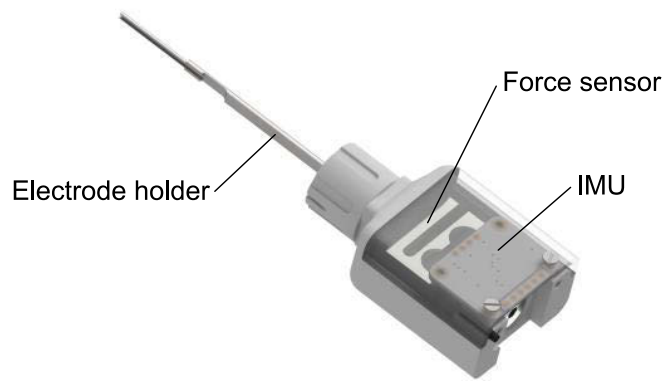

Figure 1:Rendering of the insertion tool prototype

\subsection{Software}

A software was developed for communication with the sensors, data processing and monitoring of the results. The graphical user interface was written in $\mathrm{C}++$ and designed with the framework Qt 5.12.9 (The Qt Company, Espoo, Finland). In order to enable high-frequency measurements and to maximize the responsiveness of the data visualisation, a commercial laptop with a six-core processor $(2.3 \mathrm{GHz})$ was chosen as the target platform. With the parallelisation of the monitoring and the data acquisition of both sensors, the multicore computing capacity of the processor was utilized.

\subsection{Orientation compensation}

The one dimensional force sensor measures not only externally applied forces but also forces caused by the weight of the electrode holder and the sensor itself. These forces are not constant, as they vary with orientation changes of the insertion tool relative to the gravitational field.
The measured signal $F_{S}$ can be divided into the following components:

$$
F_{S}(t)=F_{E}(t)+F_{B i a s}+F_{a}(t)
$$

The external force $F_{E}(t)$ includes all externally acting forces and is transmitted through the electrode holder. $F_{\text {Bias }}$ is a constant, orientation-independent, sensor-specific bias present in each sensor before it is set to zero. The last component $F_{a}(t)$ represents the orientation-dependent load; this is subsequentially caused by both the weight of the electrode holder and a partial weight of the sensor, summarized as $m_{E H+S}$. Therefore, $F_{a}(t)$ can be calculated with $F_{a}(t)=m_{E H+S} \cdot a_{x}(t)$. Due to the one-dimensional force sensor, only the acceleration along the measurement axis is considered in $a_{x}(t)$. Hence, $a_{x}(t)$ depends on the orientation of the insertion tool relative to the gravitational field. With changes in orientation due to manual use, this force component corrupts the measured signal.

In order to remove this error from the force signal, the following expression was derived from equation 1 :

$$
F_{E}(t)=F_{S}(t)-F_{Z P}+m_{E H+S}\left(a_{x}(t)-a_{Z P}\right)
$$

The force $F_{Z P}$ and the associated acceleration $a_{Z P}$ are determined at a random time (zero point) at the beginning of each measurement. Both factors are averaged from 100 measured values. As the measurement axes of the force sensor and the IMU are aligned, the $\mathrm{x}$-acceleration directly corresponds to $a_{x}(t)$.

The mass $m_{E H+S}$ cannot be determined with a scale because only a partial weight of the force sensor acts on its deformation body. Therefore, a calibration procedure for the determination of $m_{E H+S}$ was developed. For this purpose, the insertion tool prototype was rotated in an unloaded state with the electrode holder attached while sensor data were recorded. The rotation was done manually and aimed at a coverage of all possible orientations of the measurement axis relative to the gravitation field. Assuming that no external force was applied $\left(F_{E}(t)=0\right)$, equation 2 was solved for $m_{E H+S}$ in each measuring point and averaged for the entire recording. To avoid a division by zero, positions close to the starting point (zero point) were filtered out.

\subsection{Experimental evaluation}

For the evaluation of the developed algorithm, a $360^{\circ}$ rotation of the insertion tool prototype around an axis perpendicular to the gravitational field was performed in $45^{\circ}$ steps. The tool was attached to a $3 \mathrm{D}$ printed frame, ensuring its stable fixation 
and enabling a change of orientation around two axes in $45^{\circ}$ steps. To average out sensor noise, the mean value of the corrected force was calculated in each step based on 300 measured values over three seconds. As no external load was applied, the compensation algorithm was expected to provide an orientation-independent force of $F_{E}(t)=0 \mathrm{mN}$. Two test series each featuring orientation changes of the insertion tool prototype around a specified rotational axis were performed with a focus on residual errors. The rotational axes were defined to be perpendicular to the flat side of the tool for test series A (see Figure 2a) and perpendicular to the wide side of the tool for test series B (see Figure 2b). Since the force sensor is not rotationally symmetric along the measurement axis, this differentiation aimed at investigating the influence of the rotation axis on the measurement signal. As highlighted in Figure 3, the strain gauges are only attached to one side of the deformation body. Torques occurring around the y-axis (test series A) thus have a different impact on the measurement signal than torques around the $\mathrm{z}$-axis (test series B).
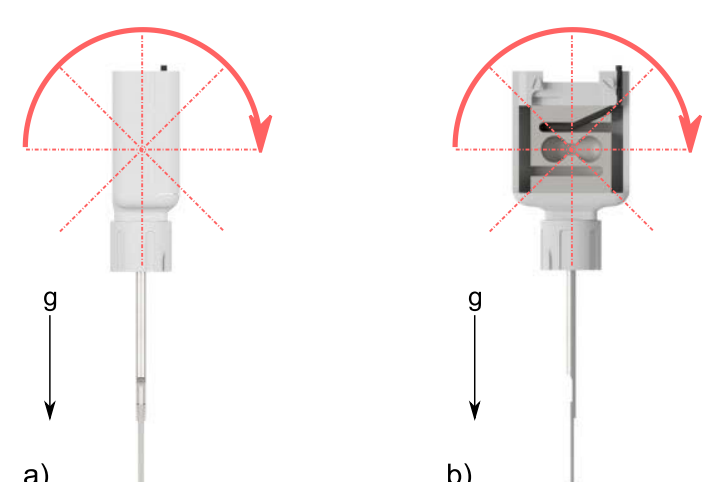

Figure 2:Different rotation axes of the insertion tool prototype with marked rotation steps. (a) Test series A. (b) Test series B.

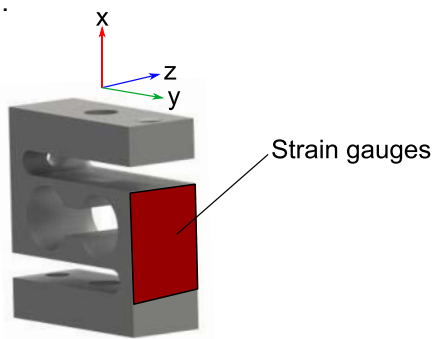

Figure 3:One dimensional force sensor KD24s. Highlighted surface indicatesplacement of strain gauges

\section{Results}

\subsection{Software}

The developed software enables the fusion of signals from the force sensor and the IMU with a frequency of up to $100 \mathrm{~Hz}$.
The corrected force is calculated and provided as feedback in a graphical user interface with an average latency of $10 \mathrm{~ms}$. In addition to online monitoring (see Figure 4), the measured and corrected forces can be saved to a CSV file.

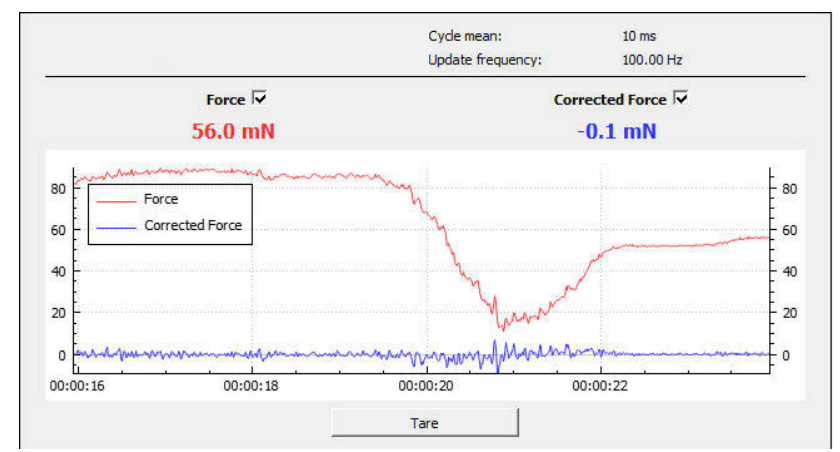

Figure 4: Graphical user interface sub-window for online monitoring of measured and corrected forces.

\subsection{Experimental evaluation}

Five recording trials were performed for each test series (total $\mathrm{n}=10$ ). The comparison of measured and corrected force demonstrates the impact of the developed algorithm (Figure 5). The mean residual errors with the corresponding standard deviation are presented in Table 1.

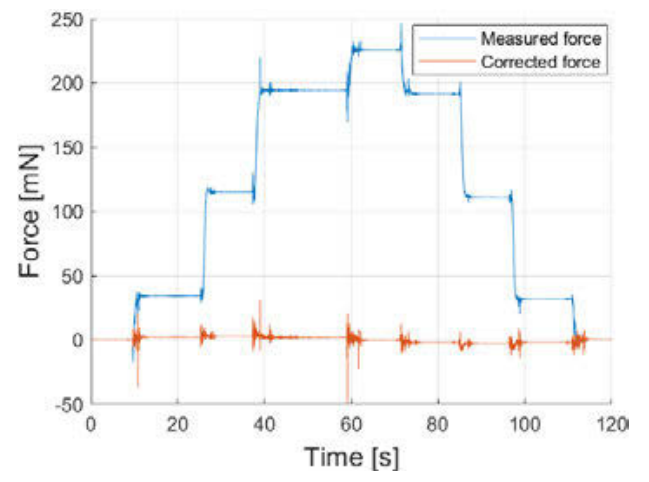

Figure 5:Exemplary comparison of measured and corrected forces for trial 5 in test series $B$.

Table 1: Mean residual error and standard deviation of corrected forces, while performing a $360^{\circ}$ rotation.

\begin{tabular}{lll}
\hline & $\begin{array}{l}\text { Mean residual } \\
\text { error }\end{array}$ & $\begin{array}{l}\text { Standard deviation } \\
\text { of the error }\end{array}$ \\
\hline Test series A & $0.399 \mathrm{mN}$ & $\pm 0.256 \mathrm{mN}$ \\
Test series B & $1.622 \mathrm{mN}$ & $\pm 1.107 \mathrm{mN}$ \\
\hline Test series A+B & $1.011 \mathrm{mN}$ & $\pm 1.008 \mathrm{mN}$ \\
\hline
\end{tabular}


The results for the individual rotation steps for test series A and $\mathrm{B}$ are shown in Figure 6 as a boxplot, to illustrate the spread of the five repetitions.

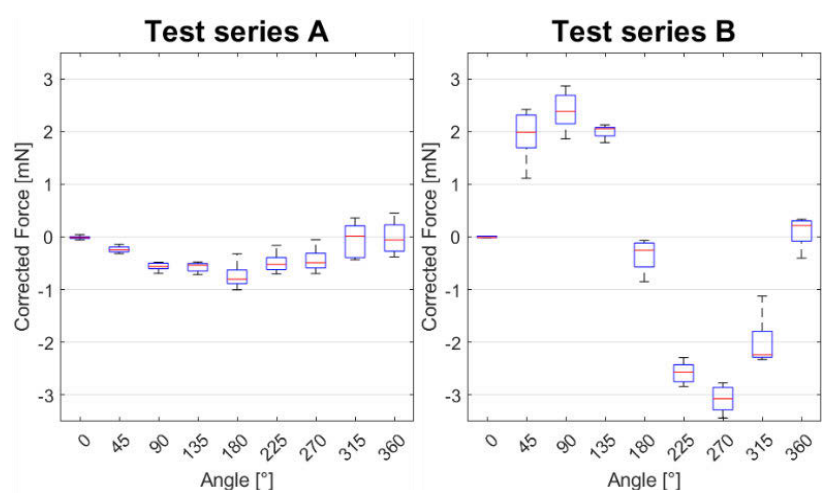

Figure 6:Boxplot of residual errors of the corrected force for each rotational step in test series $A(n=5)$ and test series $B(n=5)$.

\section{Discussion}

The proposed tool and software was developed to facilitate accurate recording of EA insertion forces during CI surgery. Such recordings can be used as surgical feedback to prevent intracochlea trauma.

Assuming the measured insertion forces with the insertion tool are comparable to forces determined in experiments with artificial cochlear models attached to a force sensor [5], insertion forces between $65 \mathrm{mN}$ and $110 \mathrm{mN}$ are expected. Measured forces at a full rotation of over $200 \mathrm{mN}$ (see Figure 5) show the considerable influence of the electrode holder and sensor weight on the measurement signal. Although such extreme angular changes are not to be expected during EA insertion, the need for a corrected signal is evident. The mean residual error in test series A of the corrected force of $0.399 \mathrm{mN}$ results in an additional measurement error of less than $1 \%$ of the expected forces and lies in an acceptable range. In contract, the mean residual error in test series $\mathrm{B}$ of $1.622 \mathrm{mN}$ is four times larger, which leaves room for improvement.

The data shown in Figure 6 indicates a systematic behaviour and one possible explanation is the S-shaped geometry of the force sensor (see Figure 3). The position of the strain gauge is assumed to be the cause for the sinusoidal course of the residual error (see Figure 6 test series B). During rotation, a torque acts on the deformation-body that is highest in the perpendicular orientation to the gravitational field and non-existent in the parallel orientation. This torque generates an additional compressive force in the strain gauge area in the first half of the rotation and a tensile force in the second half.

\section{Conclusion}

A new software for the measurement of manual EA insertion forces is presented. The software enables the correction of orientation-dependent forces in a manual force sensitive insertion tool. The high frequency of the sensor signal fusion and the calculation of the corrected force enables an almost delay-free visualisation. The combination of the insertion tool and the developed software enables the online monitoring of orientation-independent insertion forces.

The aim of future research is to analyse the remaining error and to design a computational model that eliminates it. In addition, possible strategies to provide surgeons feedback about the corrected forces will be investigated.

\section{Author Statement}

Research funding: The presented work was funded in part by the Federal Ministry of Education and Research of Germany (BMBF, grant number 13GW0367B), and in part by the Deutsche Forschungsgemeinschaft (DFG, German Research Foundation) under Germany's Excellence Strategy - EXC 2177/1 - Project ID 390895286. Conflict of interest: Authors state no conflict of interest. Informed consent: Informed consent is not applicable. Ethical approval: The conducted research is not related to either human or animals use.

\section{References}

[1] Ishii T, Takayama M, Takahashi Y. Mechanical properties of human round window, basilar and Reissner's membranes. Otolaryngol Suppl 1995;519:78-82.

[2] Mirsalehi M, Rau TS, Harbach L, et al. Insertion forcesand intracochlear trauma in temporal bone specimensimplanted with a straight atraumatic electrode array. Eur Arch Otorhinolaryngol 2017;274:2131-40.

[3] Lenarz, Thomas. Cochlear implant-state of the art. GMS current topicsin otorhinolaryngology, head and necksurgery, 2017, 16. Jg.

[4] Kaufmann, Christopher R., et al. Evaluation of insertion forces and cochlea trauma following robotics-assisted cochlear implant electrode array insertion. Otology \& Neurotology, 2020, 41. Jg., Nr. 5, S. 631-638.

[5] Zuniga, M. Geraldine, et al. The Effect of Ultra-slow Velocities on Insertion Forces: A Study Using a Highly Flexible Straight Electrode Array. Otology \& Neurotology: Official Publication of the American Otological Society, American Neurotology Society [and] European Academy of Otology and Neurotology, 2021. 